\title{
Membrane lipids and maximum lifespan in clownfish
}

\author{
Pedro F. Almaida-Pagan · Alejandro Lucas-Sanchez • \\ Antonio Martinez-Nicolas · Eva Terzibasi • Maria Angeles Rol de Lama • \\ Alessandro Cellerino $\cdot$ Pilar Mendiola $\cdot$ Jorge de Costa
}

Received: 19 February 2021 / Accepted: 18 November 2021 / Published online: 4 December 2021

(C) The Author(s) 2021

\begin{abstract}
The longevity-homeoviscous adaptation (LHA) theory of ageing states that lipid composition of cell membranes is linked to metabolic rate and lifespan, which has been widely shown in mammals and birds but not sufficiently in fish. In this study, two species of the genus Amphiprion (Amphiprion percula and Amphiprion clarkii, with estimated maximum lifespan potentials [MLSP] of 30 and 9-16 years, respectively) and the damselfish Chromis viridis (estimated MLSP of 1-2 years) were chosen
\end{abstract}

Supplementary Information The online version contains supplementary material available at https://doi. org/10.1007/s10695-021-01037-1.

P. F. Almaida-Pagan $(\bowtie) \cdot$ A. Lucas-Sanchez •

A. Martinez-Nicolas · M. A. R. de Lama - P. Mendiola ·

J. de Costa

Chronobiology Lab, Department of Physiology, Faculty

of Biology, University of Murcia, Mare Nostrum Campus,

IUIE, IMIB-Arrixaca, 30100 Murcia, Spain

e-mail: pfalmaida@um.es

P. F. Almaida-Pagan · A. Lucas-Sanchez •

A. Martinez-Nicolas · M. A. R. de Lama · P. Mendiola ·

J. de Costa

Ciber Fragilidad y Envejecimiento Saludable

(CIBERFES), Madrid, Spain

E. Terzibasi

Stazione Zoologica Anton Dohrn, Naples, Campania, Italy

A. Cellerino

Department of Neurosciences, Bio@SNS, Scuola Normale

Superiore, Pisa, Italy to test the LHA theory of ageing in a potential model of exceptional longevity. Brain, livers and samples of skeletal muscle were collected for lipid analyses and integral part in the computation of membrane peroxidation indexes (PIn) from phospholipid (PL) fractions and PL fatty acid composition. When only the two Amphiprion species were compared, results pointed to the existence of a negative correlation between membrane PIn value and maximum lifespan, well in line with the predictions from the LHA theory of ageing. Nevertheless, contradictory data were obtained when the two Amphiprion species were compared to the shorter-lived $C$. viridis. These results along with those obtained in previous studies on fish denote that the magnitude (and sometimes the direction) of the differences observed in membrane lipid composition and peroxidation index with MLSP cannot explain alone the diversity in longevity found among fishes.

Keywords Membranes · Lifespan · Fish · Lipids · Peroxidation

$\begin{array}{ll}\text { Abbreviations } \\ \text { BHT } & \text { Butylated hydroxytoluene } \\ \text { CL } & \text { Cardiolipin } \\ \text { FAME } & \begin{array}{l}\text { Fatty acid methyl esters } \\ \text { LHA }\end{array} \\ & \begin{array}{l}\text { Longevity-homeoviscous adaptation } \\ \text { theory of ageing }\end{array} \\ \text { mtROSp } & \text { Mitochondrial ROS production } \\ \text { MLSP } & \text { Maximum lifespan potential } \\ \text { PC } & \text { Phosphatidylcholine }\end{array}$




$\begin{array}{ll}\text { PE } & \text { Phosphatidylethanolamine } \\ \text { PI } & \text { Phosphatidylinositol } \\ \text { PIn } & \text { Peroxidation index } \\ \text { PL } & \text { Phospholipids } \\ \text { PS } & \text { Phosphatidylserine } \\ \text { PUFA } & \text { Polyunsaturated fatty acids } \\ \text { ROS } & \text { Reactive oxygen species } \\ \text { SM } & \text { Sphingomyelin } \\ \text { TLC } & \text { Thin layer chromatography }\end{array}$

\section{Introduction}

Reactive oxygen species (ROS) constitute the only known molecules endogenously and continuously produced by cells that have the capacity to break covalent bonds, causing damage to tissue macromolecules in biological systems (Barja 2019). Scientific evidence continues to support the mitochondrial oxygen free radical theory of ageing (Barja 2013, 2019; Miwa et al. 2014; Shen et al. 2014; López-Lluch et al. 2015; Zsurka et al. 2018) both between and within animal species. Short-lived mammals and birds have species-specific high mitochondrial ROS production (mitROSp) rates at complex I of the electron transport chain (Ku et al. 1993; Barja and Herrero 1998; Herrero and Barja 1998; Barja 2004; Lambert et al. 2007; Csiszar et al. 2012). Although ROS damage affects all cell macromolecules, lipid peroxidation is quantitatively the main oxidative process in tissues due to the high sensitivity to oxidation of polyunsaturated fatty acids (PUFA), which are essential constituents of cell membrane phospholipids (PL) (Bielski et al. 1983). Besides, lipid peroxidation is an exponential reaction chain process that generates many toxic and mutagenic by-products like the aldehydes hydroxynonenal or malondialdehyde, which can diffuse throughout the cell including the nucleus, which is poor in lipids (Chaudhary et al. 1994). Reaching the nucleus by diffusion, those aldehydes chemically react with free amino groups in DNA and could contribute to DNA damage, both in the nucleus and mitochondria.

The longevity-homeovicous adaptation (LHA) theory of ageing states that lipid composition of cell membranes (particularly that of mitochondria) is linked to metabolic rate and lifespan, which has been shown in a wide number of animal species (Pamplona et al. 1998; 2000). In comparative studies, performed on various species of mammals and birds, it has been found that species with a shorter lifespan have more unsaturated membranes than species with a longer lifespan (Pamplona 2008). Membranes with high levels of PUFA are more fluid, and the LHA theory of ageing suggests that this can enable or promote higher molecular activity of membrane proteins and, in turn, increase the metabolic activity of cells, tissues and, consequently, whole animals. At the same time, susceptibility to oxidative damage increases with the proportion of PUFA in membranes (Pamplona et al. 1998). In order to test the LHA theory of ageing in fish, where very little information is available (see Hulbert et al. 2007), we recently published a study on fishes of genus Nothobranchius (de Costa et al. 2020), which includes some of the shortest-lived vertebrates in nature (3-18 months, depending on the species) and has proved to be a remarkable system for gerontological research (Lucas-Sanchez et al. 2014; Tozzini et al. 2013). In these fishes, the longer-lived fish species have more saturated membranes and, therefore, a lower susceptibility to oxidative damage, as the LHA theory posits (de Costa et al. 2020).

On the other hand, clownfish of genus Amphiprion have been proposed as the first experimental models for long-lived vertebrates as some of its species have been reported by hobbyists (in captivity) and by researchers (in the wild) to live for more than two decades (Sahm et al. 2019). These fishes evolved a specific adaptation that allows them to live in symbiosis with sea anemones (Buston and García 2007). Under the anemone's protection, the extrinsic mortality rate of these fish is low, and, following the standard evolutionary theories of ageing, low extrinsic mortality conditions lead to the evolution of slow senescence and increased lifespan (Mariscal 1970; Aldenhoven 1986; Eckert 1987; Elliot et al. 1995; Buston 2003; Blanco and Sherman 2005).

In this study, two species of the genus Amphiprion (Amphiprion percula and Amphiprion clarkii, with estimated maximum lifespan potentials [MLSP] in the wild of 30 and 9-16 years, respectively) (Moyer 1986; Buston and García 2007; Sahm et al. 2019) were studied along with the damselfish Chromis viridis, to test how the LHA theory of ageing applies to this fish group. $C$. viridis belong to the non-symbiotic sister-taxon of the Amphiprion genus, share with them general traits linked to their life in nature and show an interesting relationship with branching corals (Garcia-Herrera et al. 2017). However, despite 
the presence of a favourable microhabitat, $C$. viridis are predated by a wide range of generalist predator species, which has been suggested to be linked to a higher mortality rate (Hixon and Carr 1997; Sahm et al. 2019) and shorter lifespan (estimated MLSP of 1-2 years) (Wantiez and Thollot 2001; Sahm et al. 2019).

\section{Methods}

Animal housing and sampling

Young adults (taken just after attaining adult size and sexual maturation, which is 2-3 years approx., for the Amphiprion species and 1 year for the damselfish) of Amphiprion percula (total length, $45.2 \pm 1.2 \mathrm{~mm}$; total weight, $1.6 \pm 0.4 \mathrm{~g} ; n=12$ ), Amphiprion clarkii $\left(L_{\mathrm{T}}, 46.4 \pm 5.1 \mathrm{~mm} ; W_{\mathrm{T}}, 2.3 \pm 1.19 \mathrm{~g} ; n=12\right)$ and Chromis viridis $\left(L_{\mathrm{T}}, 43.0 \pm 1.6 \mathrm{~mm} ; W_{\mathrm{T}}, 1.3 \pm 0.4 \mathrm{~g}\right.$; $n=12$ ) (Perciformes, Pomacentridae) were used for the present study (Fig. 1). Fishes were acquired from local dealers and subjected to acclimation during 1 month in the facilities of the Marine Aquarium of the University of Murcia. Fish were kept in groups in a recirculating system (one tank per species, stock density of $0.30 \mathrm{~g} / \mathrm{L}$ ) under exactly the same

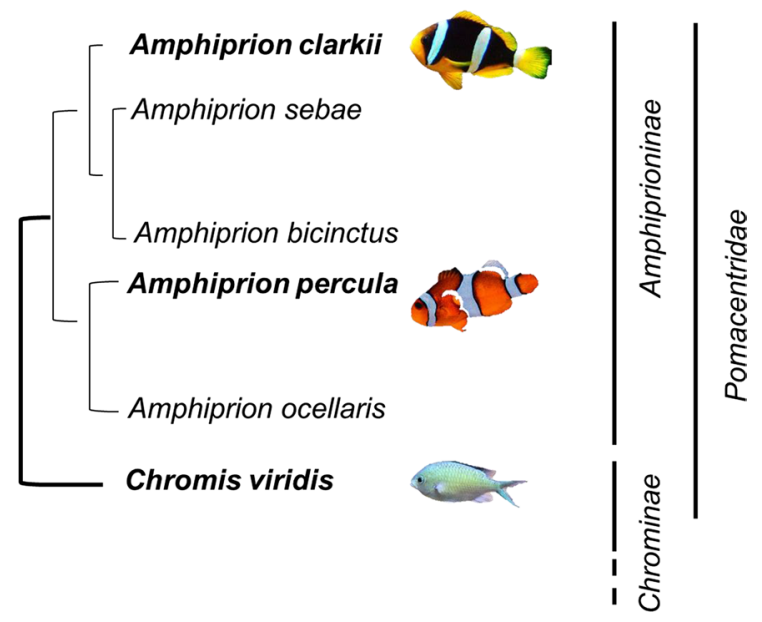

Fig. 1 Nucleotide-based phylogeny of the analysed fish species as estimated in a previous study (Sahm et al. 2019). Chromis viridis represents the non-symbiotic sister-taxon of the Amphiprion genus. A. percula photo by Dylan McLeod on Unsplash. A. clarkii picture by Citron, and $C$. viridis photo by Ben Lancaster (both taken from Wikipedia) conditions (temperature, $27 \pm 1{ }^{\circ} \mathrm{C}$; salinity, $24 \pm 1$; $\mathrm{pH}=8 \pm 0.2$; dissolved oxygen, $6.5 \pm 0.2 \mathrm{mg} / \mathrm{L}$ ) and fed ad libitum twice a day a standard diet to match their requirements (Mysis shrimp, enriched Artemia nauplii and red plankton).

Fishes were euthanized by exposure to the anaesthetic tricaine methanesulfonate (MS222, $200 \mathrm{mg} / \mathrm{L}$ ) for 10 min following the cessation of gill movement. Brain, livers and samples of skeletal muscle (collected above the lateral line, between the dorsal fin and the caudal fin) were collected, pooled and homogenized to produce quadruplicate samples (4 pools of 3 fish) for lipid analyses. Fresh samples were placed directly in chloroform/methanol $(2: 1, \mathrm{v} / \mathrm{v})$ containing $0.01 \%$ (w/v) butylated hydroxytoluene as antioxidant and subjected to total lipid extraction (Folch et al. 1957).

Lipid extraction and phospholipid class composition

Briefly, fish samples ( $2 \mathrm{~g}$ of skeletal muscle and $0.5 \mathrm{~g}$ of liver and brain) were homogenized in $20 \mathrm{~mL}$ of ice-cold chloroform/methanol followed by the addition of $5 \mathrm{~mL}$ of $0.88 \%$ (w/v) $\mathrm{KCl}$, mixing, and layers allowed to separate on ice for $1 \mathrm{~h}$. The upper aqueous layer was aspirated, and the lower organic layer was evaporated under a stream of oxygen-free nitrogen. All lipid extracts were stored at $-20{ }^{\circ} \mathrm{C}$ under a $\mathrm{N}_{2}$ atmosphere prior to analysis. PL classes were separated by high-performance thin-layer chromatography using $10-\times 10-\mathrm{cm}$ silica gel plates (VWR, Lutterworth, England) and methyl acetate/isopropanol/chloroform/methanol/0.25\% (w/v) $\mathrm{KCl}$ (25:25:25:10:9, by volume) as solvent system (Olsen and Henderson 1989). The lipid classes were visualized by charring at $160{ }^{\circ} \mathrm{C}$ for $15 \mathrm{~min}$ after spraying with $3 \%$ $(\mathrm{w} / \mathrm{v})$ aqueous cupric acetate containing $8 \%(\mathrm{v} / \mathrm{v})$ phosphoric acid and quantified by visible densitometry using Image Scanner II (Amersham Biosciences, UK). Scanned images were recorded automatically and analysed by computer using IQ-Image Quant TL 8.1 software (GE Healthcare Bio-Sciences AB, Sweden).

\section{Phospholipid fatty acid composition}

Individual phospholipid (PL) classes from tissue's total lipid extract were separated by preparative-TLC, using silica gel plates $(20 \times 20 \mathrm{~cm})(\mathrm{VWR})$ and the 
solvent system as above. Individual PL classes were identified by comparison with known standards after spraying with $1 \%(\mathrm{w} / \mathrm{v}) 2^{\prime}, 7^{\prime}$-dichlorofluorescein in 97\% (v/v) methanol containing $0.05 \%$ (w/v) BHT, and visualization under UV light. Each PL class was scraped from the plate into a test tube and subjected directly (on silica) to acid-catalysed transmethylation at $50{ }^{\circ} \mathrm{C}$ overnight following addition of $2 \mathrm{~mL}$ of $1 \%$ $(\mathrm{v} / \mathrm{v})$ sulphuric acid in methanol in order to prepare fatty acid methyl esters (FAME) (Christie 2003). FAME were separated and quantified by gas-liquid chromatography. For this, a Hewlett-Packard 5890 gas chromatograph with a capillary column (SPTH2560, SUPELCO, $100 \mathrm{~m} \times 0.25 \mathrm{~mm}$ I.D., $0.20-\mu \mathrm{m}$ film thickness) was used. The oven temperature, held at an initial value of $140{ }^{\circ} \mathrm{C}$ for $5 \mathrm{~min}$, was increased at a rate of $4{ }^{\circ} \mathrm{C}$ per min to $230{ }^{\circ} \mathrm{C}$, then further increased at a rate of $1{ }^{\circ} \mathrm{C}$ per min to $240{ }^{\circ} \mathrm{C}$ and finally held at that temperature for $6 \mathrm{~min}$. The injector and flame ionization detector were set at $250{ }^{\circ} \mathrm{C}$. Helium at a pressure of $290 \mathrm{kPa}$ was used as carrier gas. Peaks were identified by comparing their retention times with appropriate FAME standards purchased from Sigma Chemical Company (St. Louis, MO, USA). Individual fatty acid concentrations were expressed as percentages of the total content.

\section{Indexes and statistical analysis}

The peroxidation index (PIn) was used as an estimate of every single PL susceptibility to oxidation and was calculated using the formula: PIn $=0.025 \times($ percentage of monoenoics $)+1 \times$ (percentage of dienoics $)+2 \times($ percentage of trienoics $)+4 \times($ percentage of tetraenoics $)+6 \times$ (percentage of pentaenoics) $+8 \times$ (percentage of hexaenoics) (Witting and Horwitt 1964). PIn for total PL was calculated as the weighted sum of each PL's PIn (Total PL's PIn $=\mathrm{p} 1$ $\mathrm{PIn}_{\mathrm{PC}}+\mathrm{p} 2 \mathrm{PIn}_{\mathrm{PE}}+\mathrm{p} 3 \mathrm{PIn}_{\mathrm{CL}}+\mathrm{p} 4 \mathrm{PIn}_{\mathrm{PS}}+\mathrm{p} 5 \mathrm{PIn}_{\mathrm{PI}}+\mathrm{p} 6$ $\mathrm{PIn}_{\mathrm{SM}}$, where $\mathrm{p} 1-6$ are the relative contents of each PL class). Results are presented as mean $\pm \operatorname{SD}(n=4)$. Where necessary, data were arc-sin transformed before further statistical analysis. A one-way analysis of variance (ANOVA) was used to compare individual PL proportions, single fatty acids or groups of fatty acids and indexes between tissues for each species and, then, to compare individual PL proportions, single fatty acids or groups of fatty acids and indexes between species for each tissue. Tukey's post hoc test was used for multiple comparisons when pertinent, and data's homogeneity of variances was checked by the Levene's test. $P<0.05$ was considered to be statistically different. Statistical analyses were performed using SPSS, version 22.0 (SPSS Inc., Chicago, IL).

\section{Results}

Phospholipid class composition

Percentages of the main phospholipid (PL) classes that integrate tissue membranes from Amphiprion percula, A. clarkii and Chromis viridis are represented in Fig. 2. Fish liver, skeletal muscle and brain showed different membrane PL compositions within each species. When the three tissues' PL composition was compared for each fish species using a one-way ANOVA, liver membranes showed a significantly higher sphingomyelin (SM) relative content $([F(2,9=135.949, P<0.001]$ for $A$. percula, $[F(2,9)=73.298, P<0.001]$ for $A$. clarkii and $[F(2,9)=10.777, P=0.005]$ for $C$. viridis) and a higher cardiolipin (CL) proportion than those from skeletal muscle and brain in the three species $\quad([F(2,9=4.823, \quad P=0.048], \quad[F(2,9)=35.414$, $P<0.001]$ and $[F(2,9)=43.775, P<0.001]$ for $A$. percula, A. clarkii and $C$. viridis, respectively). Besides, liver membranes had a lower phoshatidylserine (PS) relative content $([F(2,9=52.876, P<0.001]$, $[F(2,9)=34.824, \quad P<0.001]$ and $[F(2,9)=78.188$, $P<0.001]$ for $A$. percula, $A$. clarkii and $C$. viridis, respectively). On the other hand, brain membranes showed higher PS and phosphatidylethanolamine (PE) $([F(2,9=16.222, P=0.002],[F(2,9)=41.046$, $P<0.001]$ and $[F(2,9)=17.070, P=0.001]$ for $A$. percula, A. clarkii and $C$. viridis, respectively) and lower phosphatidylinositol (PI) ([F(2,9=48.874, $P<0.001]$, $[F(2,9)=33.797, \quad P<0.001]$ and $[F(2,9)=57.707$, $P<0.001]$ for $A$. percula, A. clarkii and $C$. viridis, respectively) and CL than those from liver and skeletal muscle in the three fish species. Regarding interspecies comparisons, no significant differences in PL class percentages were found in liver membranes among Amphiprion percula, A. clarkii and Chromis clarkii $([F(2,9)=4.957, P=0.054],[F(2,9)=3.093$, $P=0.119], \quad[F(2,9)=0.254, \quad P=0.784]$, $[F(2,9)=4.560, \quad P=0.062], \quad[F(2,9)=2.463$, $P=0.166]$ and $F(2,9)=1.190, P=0.367]$ for $\mathrm{SM}$, 
Fig. 2 Phospholipid class composition (percentage of total phospholipids) of membranes isolated from three different tissues (liver, skeletal muscle and brain) of Amphiprion percula (A), Amphiprion clarkii (B) and Chromis viridis $(\mathbf{C})$. Results are mean $\pm \operatorname{SD}(n=4)$.

Superscript letters indicate the existence of statistical differences among tissues for each phospholipid class as determined by a one-way ANOVA and Tukey's post hoc test ("b" indicates a statically higher value than "a" for the same PL class; $P<0.05)$. CL cardiolipin, n.s. non-significant, $\mathrm{PC}$ phosphatidylcholine, PE phosphatidylethanolamine, PI phosphatidylinositol, PS phosphatidylserine, SM sphingomyelin
A)

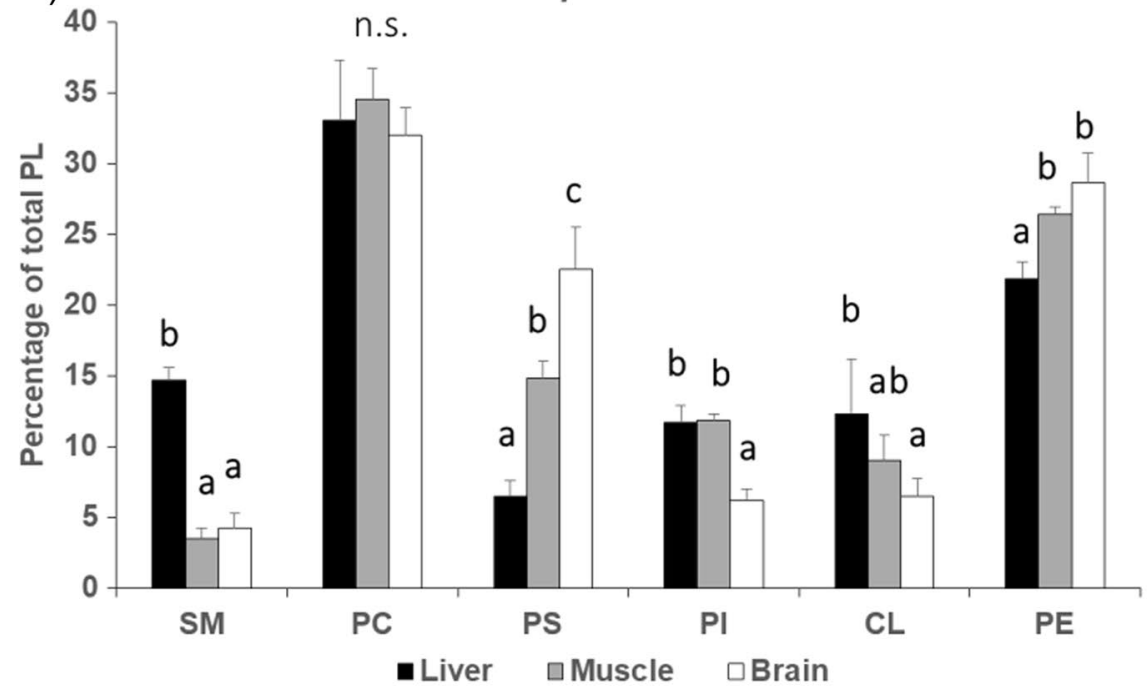

B)

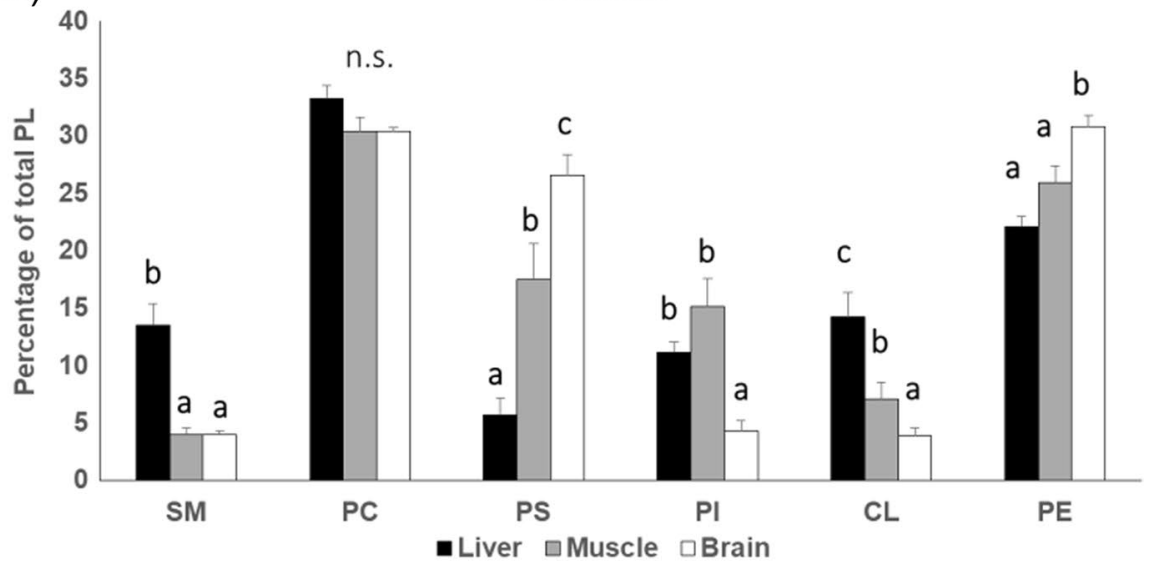

C)

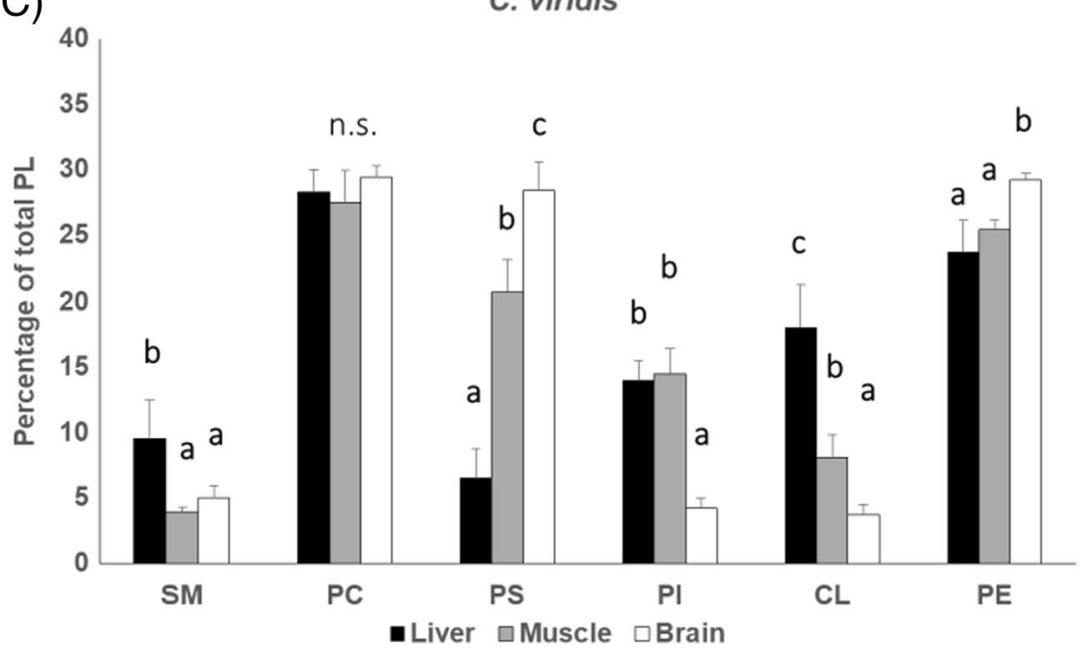


PC, PI, PS, CL and PE, respectively) (Fig. 3A). Skeletal muscle from the coral reef damselfish $C$. viridis had higher relative contents of PS $[\mathrm{F}(2,9)=11.390$, $P=0.005]$ and lower of PC $[F(2,9)=10.753$, $P=0.005]$ than $A$. percula (Fig. $3 \mathrm{~B}$ ), and brain membranes from $A$. percula showed a higher relative content in PI $[F(2,9)=7.304, P=0.016]$ and CL $[F(2,9)=9.762, P=0.007]$ and lower of PS $[F(2,9)=6.147, P=0.024]$ than $C$. viridis (Fig. 3C).

Fatty acid compositions and peroxidation index values of individual PLs

Figures 4, 5 and 6 show the fatty acid composition and peroxidation index (PIn) values of tissue membranes (liver, skeletal muscle and brain, respectively) from young adult Amphiprion percula, Amphiprion clarkii and Chromis viridis kept under equal temperature and rearing conditions and fed the same diet. For clarity reasons, pie charts in every figure represent only the groups of fatty acids (sum of saturated, sum of monounsaturated, sum of n-6 polyunsaturated and sum of n-3 polyunsaturated) and the most representative fatty acids within each group. Only the four more significant PL for each tissue (the three more abundant PL plus CL, the sum of which was at least $81.8 \%$ of total PL) are presented. The complete fatty acid composition and indexes for every PL class and tissue for the three experimental fish species are included as Supplementary material (Supp. Tables 1-18).

Regarding fish liver, the peroxidation index (PIn) was significantly lower in $C$. viridis than in the two Amphiprion species for all PL classes $([F(2,9)=23.775, \quad P=0.001], \quad[F(2,9)=12.920$, $P=0.004], \quad[F(2,9)=9.670, \quad P=0.01]$, $[F(2,9)=11.652, \quad P=0.009], \quad[F(2,9)=71.658$. $P<0.001]$ for PE, CL, PI, PS and PC, respectively), except for sphingomyelin (SM) $[F(2,9)=40.068$, $P=0.001]$, in which PIn was higher in $C$. viridis $(\mathrm{PIn}=52.1 \pm 1.3)$ than in the other two fish species (PIn $=31.7 \pm 2.5$ and $45.2 \pm 3.2$ for A. percula and A. clarkii, respectively) (Fig. 4, Supp. Tables 1-6). PIn values for $A$. clarkii were higher than those for A. percula for PC $(279.4 \pm 13.5$ vs. $180.3 \pm 24.1)$, PE $(380.7 \pm 22.6$ vs. $340.7 \pm 11.3)$ and SM. PIn value for total PL was significantly lower in $C$. viridis $\quad(171.5 \pm 3.7) \quad[F(2,9)=126.387, \quad P<0.001]$. Regarding the two Amphiprion species, PIn of total PL was higher in A. clarkii $(255.4 \pm 9.7)$ than in
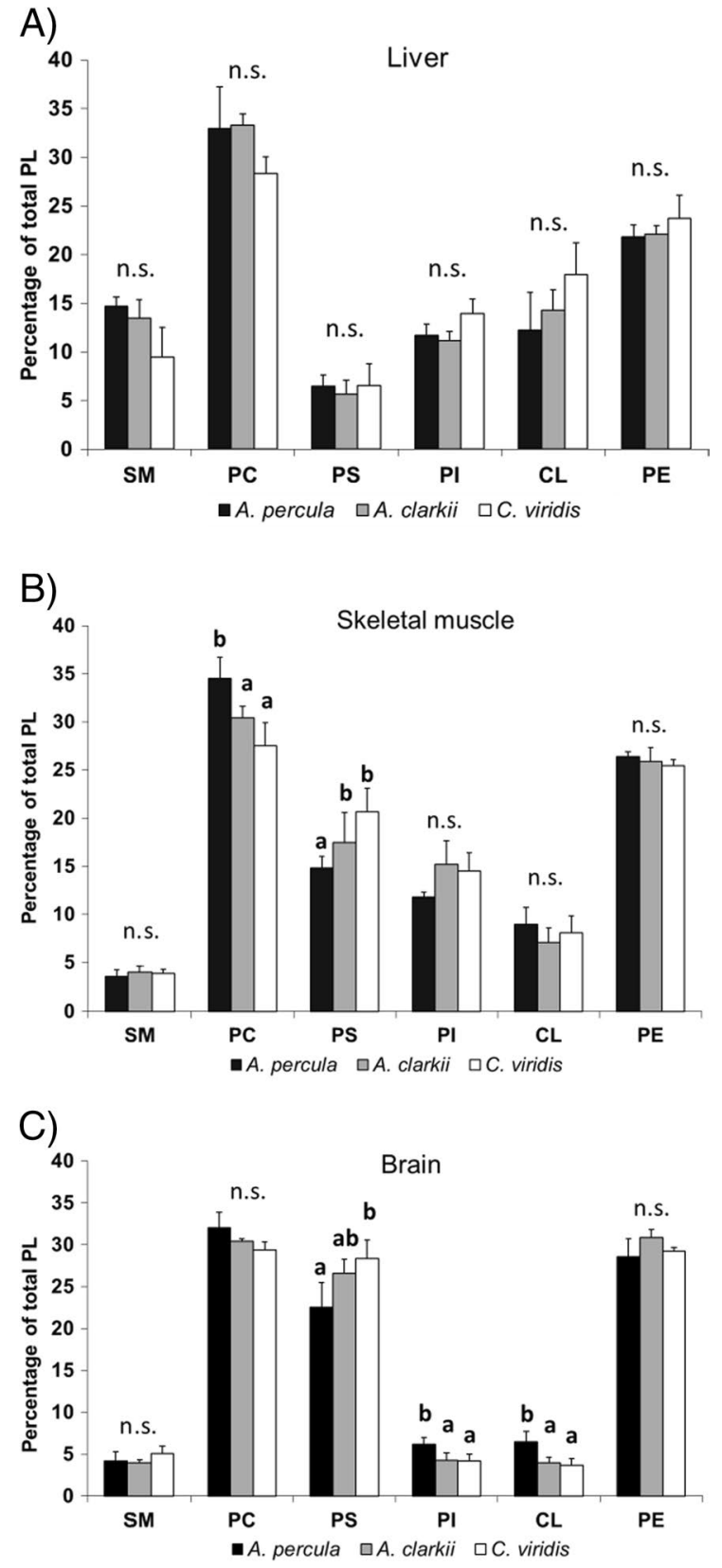

Fig. 3 Phospholipid class composition (percentage of total phospholipids) of membranes isolated from liver (A), skeletal muscle (B) and brain (C) of young adult Amphiprion percula, Amphiprion clarkii and Chromis viridis. Results are mean \pm SD $(n=4)$. Superscript letters indicate the existence of statistical differences among fish species for each phospholipid class as determined by a one-way ANOVA and Tukey's post hoc test ("b" indicates a statically higher value than "a" for the same PL class; $P<0.05)$. CL cardiolipin, n.s. non-significant, PC phosphatidylcholine, PE phosphatidylethanolamine, PI phosphatidylinositol, PS phosphatidylserine, SM sphingomyelin 
Fig. 4 Phospholipid fatty acid composition of liver membranes from young adult Amphiprion percula, Amphiprion clarkii and Chromis viridis. Each segment of the pie chart represents the following fatty acids (clockwise order): saturated (black: 16:0 and ¿saturated), monounsaturated (dark grey: 18:1n-9 and $\Sigma$ monounsaturated), n-6 polyunsaturated (light grey: 18:2, 20:4 and $\Sigma \mathrm{n}-6$ PUFA), n-3 polyunsaturated (white: 18:3, 20:5, 22:6 and nn-3 PUFA). Right column graphs present peroxidation index (PIn) values of each PL class for the three fish species. B) PIn values for membrane total PL from liver of the three fish species. Results shown in PIn graphs are mean \pm SD $(n=4)$. Superscript letters mean statistical differences among fish species for PIn values as determined by a one-way ANOVA and Tukey $t$ test ("b" indicates a statically higher value than "a" for the same PL class; $P<0.05)$. CL cardiolipin, PC phosphatidylcholine, $\mathrm{PE}$ phosphatidylethanolamine, PI phosphatidylinositol, PS phosphatidylserine, SM sphingomyelin
A)

A. percula

A. clarkii

C. viridis

PC
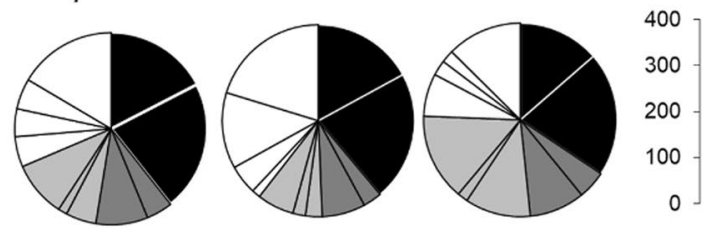

Peroxidation Index
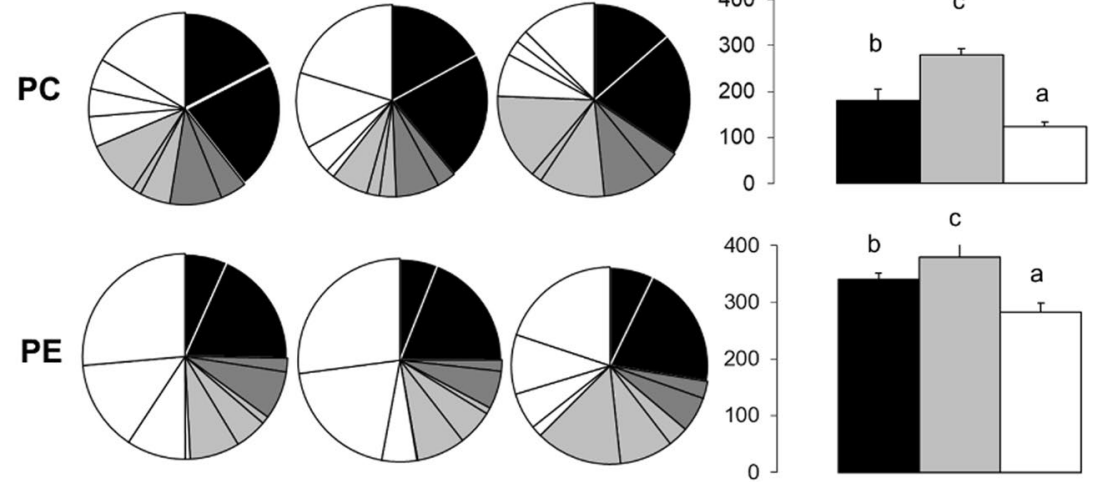

SM
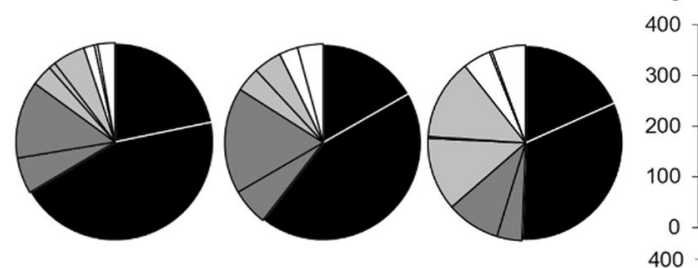

400
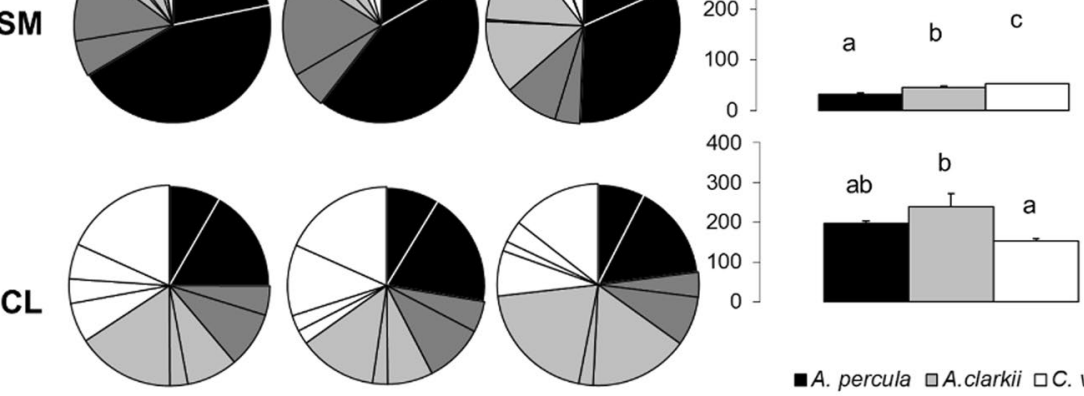

A. percula $\square$ A.clarkii $\square$ C. viridis

B)

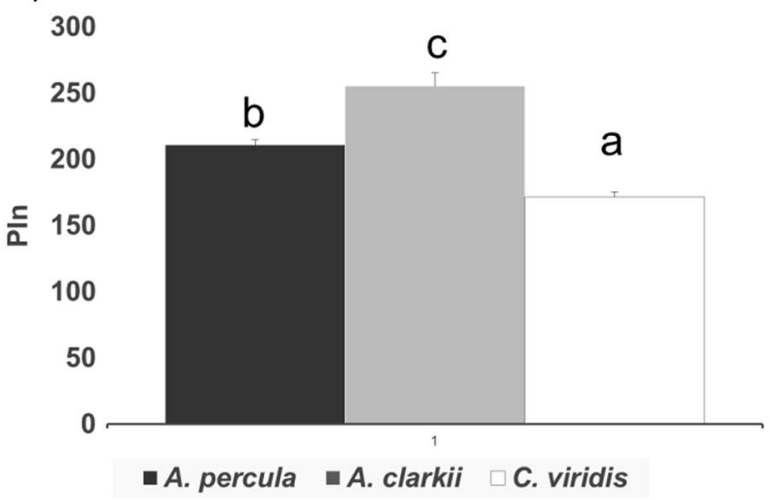

A. percula $(210.9 \pm 4.0)$. Liver membranes from C. viridis showed a lower content in $\mathrm{n}-3$ polyunsaturated fatty acids (PUFA) in PC $(22.0 \pm 1.2)$, PE $(33.0 \pm 1.5)$ and PS $(26.0 \pm 1.6)$ and higher in SM $(9.3 \pm 0.8)$ when compared with A. percula $(29.1 \pm 4.0,43.7 \pm 1.1,35.8 \pm 1.9$ and $4.3 \pm 1.3$ for PC, PE, PS and SM, respectively) and A. clarkii $(36.4 \pm 1.8, \quad 45.3 \pm 6.7, \quad 37.3 \pm 4.9$ and $6.0 \pm 2.4)$ $([F(2,9)=24.168, P<0.001]$ for PC, $[F(2,9)=8.154$,
$P=0.019]$ for PE, [ $F(2,9)=11.418, P=0.009]$ for PS and $[F(2,9)=6.763, P=0.045]$ for $\mathrm{SM})$.

There were significant differences in PIn values for skeletal muscle membranes among the experimental fish species for PC $[F(2,9)=43.240$, $P<0.001]$, PS $[F(2,9)=12.086, P=0.004]$ and PI $[F(2,9)=50.998, P<0.001]$ (Fig. 5, Supp. Tables 7-12). PIn values for PS and PI were lower in A. percula $(196.5 \pm 16.9$ and $242.1 \pm 7.5)$ than in 
Fig. 5 Phospholipid fatty acid composition of skeletal muscle membranes from young adult Amphiprion percula, Amphiprion clarkii and Chromis viridis. Each segment of the pie chart represents the following fatty acids (clockwise order): saturated (black: 16:0 and ¿saturated), monounsaturated (dark grey: 18:1n-9 and $\Sigma$ monounsaturated), n-6 polyunsaturated (light grey: 18:2, 20:4 and $\Sigma \mathrm{n}-6$ PUFA), $\mathrm{n}-3$ polyunsaturated (white: 18:3, 20:5, 22:6 and $\Sigma$ n-3 PUFA). Right column graphs present peroxidation index (PIn) values of each PL class for the three fish species. B) PIn values for membrane total PL from skeletal muscle of the three fish species. Results shown in PIn graphs are mean \pm SD $(n=4)$. Superscript letters mean statistical differences among fish species for PIn values as determined by a one-way ANOVA and Tukey $t$ test ("b" indicates a statically higher value than "a" for the same PL class; $P<0.05)$. CL cardiolipin, n.s. non-significant, $\mathrm{PC}$ phosphatidylcholine, $\mathrm{PE}$ phosphatidylethanolamine, PI phosphatidylinositol, PS phosphatidylserine, SM sphingomyelin
A)

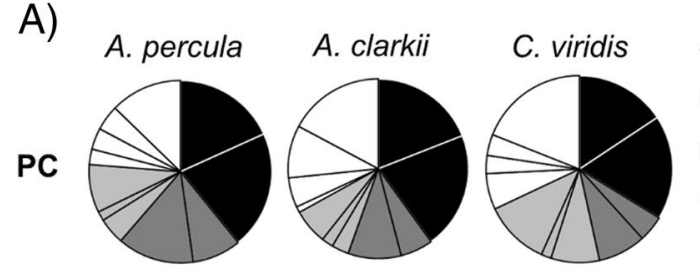

Peroxidation Index
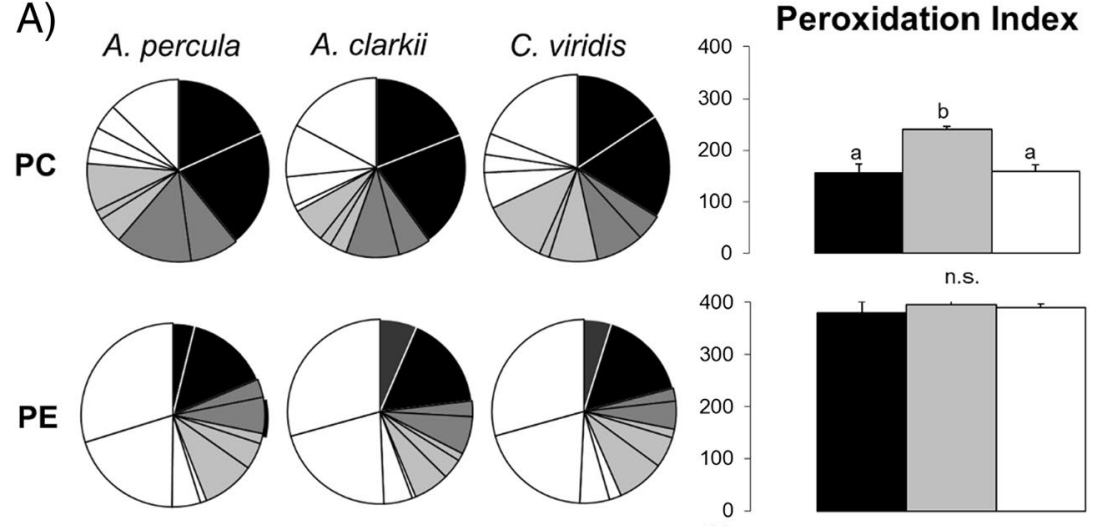

PS
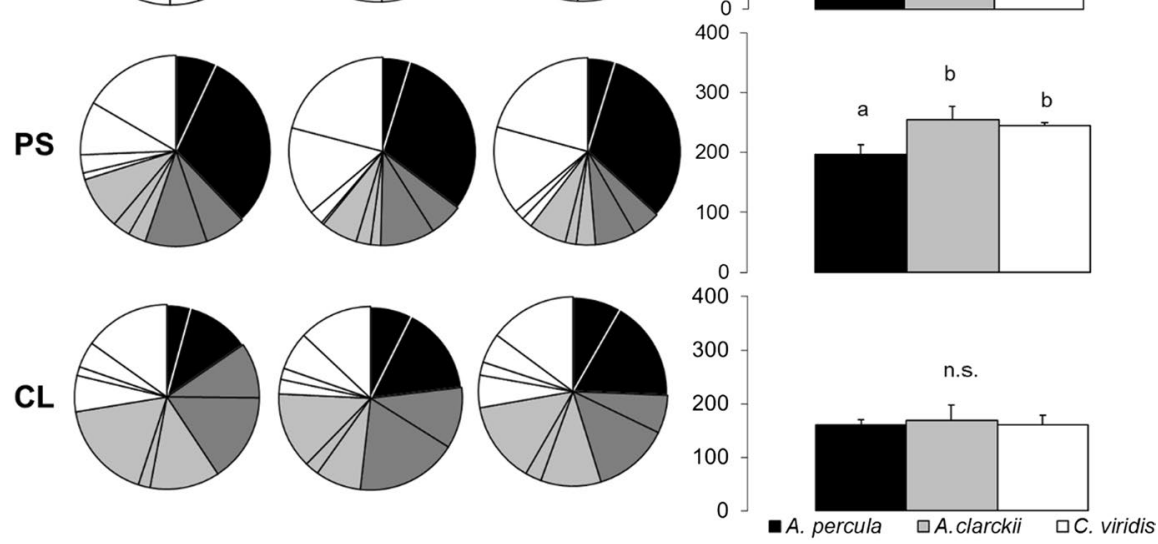

B)

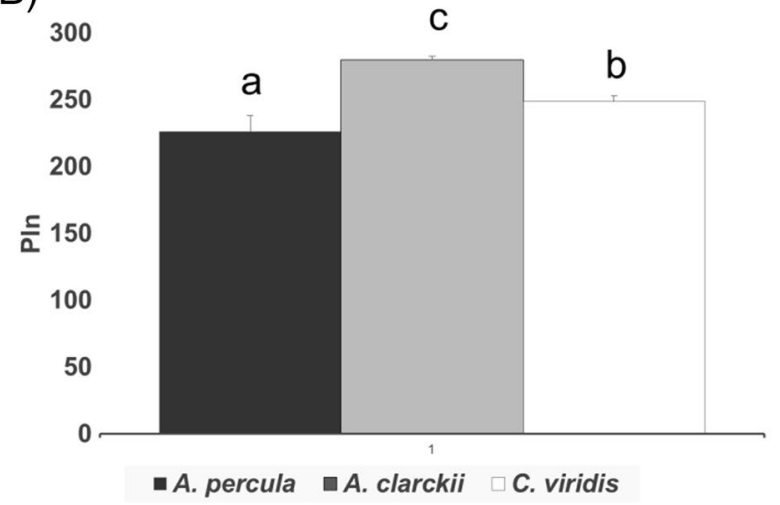

A. clarkii $(255.1 \pm 21.9$ and $294.0 \pm 6.2)$ and C. viridis $(244.4 \pm 6.1$ and $282.6 \pm 7.2)$, while PC PIn was higher in A. clarkii $(240.4 \pm 5.1)$ compared to $A$. percula $(155.9 \pm 16.8)$ and $C$. viridis $(158.5 \pm 13.6)$. Regarding the two Amphiprion species, PIn values for PC, PS and PI were lower in A. percula compared to A. clarkii. Total PL PIn value was significantly higher in $A$. clarkii $(279.7 \pm 2.7)$ than in $C$. viridis $(248.8 \pm 4.0)$ and $A$. percula $(226.5 \pm 11.4)$ $[F(2,9)=46.666, \quad P<0.001]$. Skeletal muscle membranes from A. percula showed a lower n-3 PUFA content in PC $(22.8 \pm 1.9)$, PS $(24.8 \pm 1.9)$ and PI $(22.2 \pm 1.4)$ than A. clarkii $(31.8 \pm 0.3$, $31.2 \pm 4.0$ and $30.5 \pm 1.8)$ and $C$. viridis $(26.9 \pm 2.1$, $31.3 \pm 0.8$ and $36.0 \pm 1.3) \quad([F(2,9)=19.707$, $P<0.001]$ for PC, $[F(2,9)=6.497, P=0.021]$ for $\mathrm{PS}$ and $[F(2,9)=70.368, P<0.001])$.

Regarding fish brain, PIn values showed no significant differences among fish species for any of the four 
Fig. 6 Phospholipid fatty acid composition of brain membranes from young adult Amphiprion percula, Amphiprion clarkii and Chromis viridis. Each segment of the pie chart represents the following fatty acids (clockwise order): saturated (black: 16:0 and ¿saturated), monounsaturated (dark grey: 18:1n-9 and $\Sigma$ monounsaturated), n-6 polyunsaturated (light grey: 18:2, 20:4 and $\Sigma \mathrm{n}-6$ PUFA), $\mathrm{n}-3$ polyunsaturated (white: 18:3, 20:5, 22:6 and इn-3 PUFA). Right column graphs present peroxidation index (PIn) values of each PL class for the three fish species. B) PIn values for membrane total PL from brain of the three fish species. Results shown in PIn graphs are mean \pm SD $(n=4)$. Superscript letters mean statistical differences among fish species for PIn values as determined by a one-way ANOVA and Tukey $t$ test ("b" indicates a statically higher value than "a" for the same PL class; $P<0.05)$. CL cardiolipin, n.s. non-significant, $\mathrm{PC}$ phosphatidylcholine, PE phosphatidylethanolamine, PI phosphatidylinositol, PS phosphatidylserine, SM sphingomyelin
A)
A. percula
A. clarkii
C. viridis

Peroxidation Index

PC
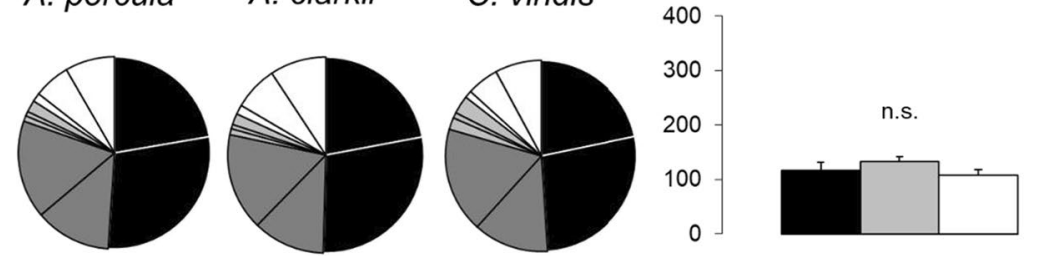

n.s.
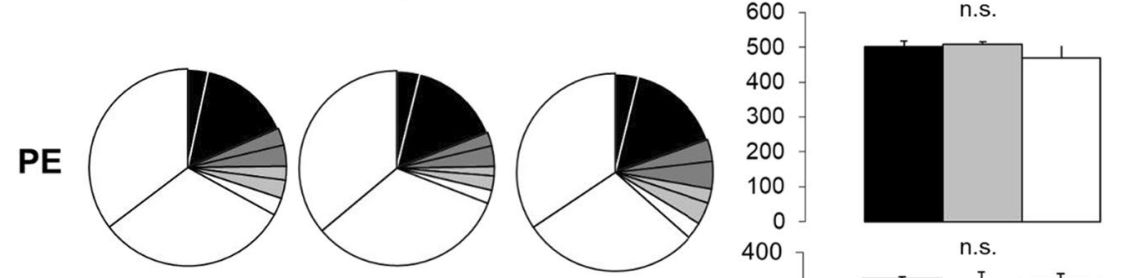

400

300

PS
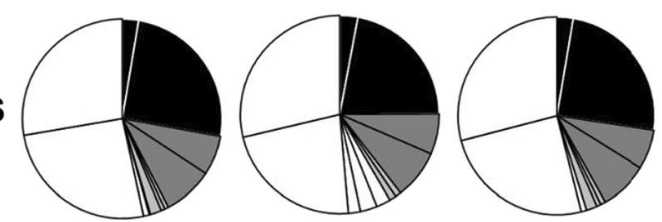

CL
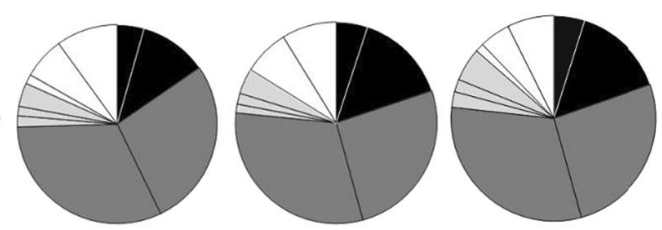

\section{100
0
400 \\ 400
300}

$\begin{gathered}200 \\ 100 \\ 0\end{gathered}-$

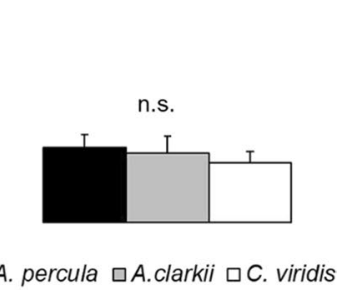

A. percula $\square$ A.clarkii $\square$ C. viridis

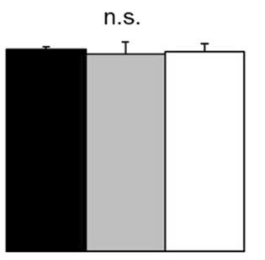

B)

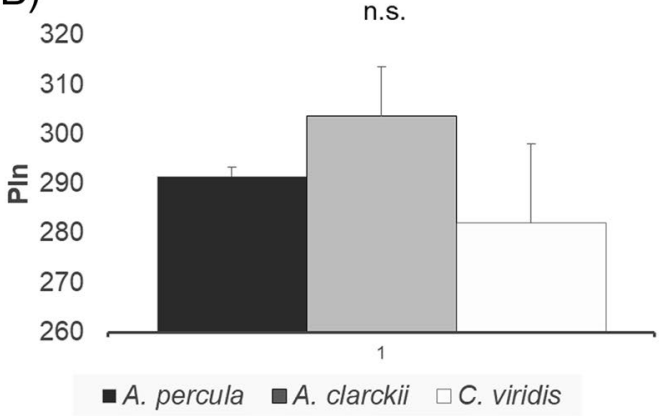

B) 
were richer in sphingomyelin (SM) and cardiolipin (CL), those from brain had a higher content in phophatidylserine (PS) and phosphatidylethanolamine (PE) than the other tissues. As discussed below, a high PE content may play a protective role from the ageing-associated damage caused by ROS in this tissue in particular (Feng et al. 2014). This is interesting as brain had the most unsaturated membranes compared to liver and skeletal muscle within each species, which render them more sensitive to oxidative damage (Hulbert et al. 2006b).

Tissue membranes showed significant differences in PL proportions among the three species. PS content was statistically higher in skeletal muscle and brain from the shorter-lived $C$. viridis (estimated MLSP $=1-2$ years) compared to the two Amphiprion species (estimated MLSP of 30 and 9-16 for A. percula and A. clarkii, respectively). This is in accordance with that observed in a previous study where three fish species of the short-lived annual genus Nothobranchius (Nothobranchius korthausae, Nothobranchius rachovii, Nothobranchius guentheri) with different MLSP (MLSP=80, 63 and 53 weeks, respectively) and the longer-lived outgroup species Aphyosemion australe (MLSP $=156$ weeks) were studied to test whether they conform to the predictions of the LHA theory of ageing (de Costa et al. 2020). A negative correlation between fish MLSP and PS content from cell membranes was also found in the Nothobranchius study and suggested to be linked to PS decarboxylation. PS descarboxylation leads to an increase in phosphatidylethanolamine (PE) intracellular levels, which was also found in the longer-lived Nothobranchius species and in A. australe. Since the abundance of PE positively regulates autophagy, regarded as one of the major cytoprotective mechanisms during ageing (Feng et al. 2014), both a lower content of PS and higher of PE in cell membranes could indicate that this mechanism is operating to protect cells and tissues from the ageing-associated damage caused by ROS (de Costa et al. 2020). Nevertheless, no statistical differences among Amphiprion species and $C$. viridis in PE levels were found in any tissue's membranes.

Regarding membrane PL fatty acid composition of fish tissues from the two Amphiprion species, A. percula had less unsaturated membranes and, thus, lower peroxidation levels in liver and skeletal muscle (not statistical differences were found for brain) than
A. clarkii, which has an estimated MLSP of half that of A. percula (9-16 vs. 30 years). This occurred at the level of the main PL classes from the two tissues, as it was shown in whole body of the Nothobranchius species previously studied (de Costa et al. 2020) and supports the longevity-homeovicous adaptation (LHA) theory of ageing (Pamplona et al. 1998, 2000, 2002; Naudí et al. 2013). In previous studies performed by Hulbert et al., a strong inverse relationship was found between peroxidation index (PIn) of liver mitochondrial PLs and skeletal muscle PLs and MLSP of mammals (Hulbert 2005; Hulbert et al. 2006a) (see Fig. 7 in Hulbert et al., 2007). The relationship between liver mitochondrial phospholipid PIn of mammals is proportional to their MLSP ${ }^{-0.40}$, which means that a $24 \%$ decrease in their peroxidative susceptibility is associated with every doubling of MSLP. For skeletal muscle membranes, the corresponding value is that a $19 \%$ decrease in peroxidative susceptibility is associated with every doubling of MLSP in mammals (muscle PIn is proportional to $\mathrm{MLSP}^{-0.30}$ ). Our data reflected a PIn reduction of $17.4 \%$ and $19 \%$ in liver and skeletal muscle membrane phospholipids, respectively, which is quite close to that observed in mammals.

When membrane PL fatty acid composition from tissues of the two Amphiprion species was compared with that of $C$. viridis, we found that $C$. viridis membranes had generally a lower PIn value than that from one (in skeletal muscle) or the two Amphiprion species (in liver), this being in contradiction with the LHA theory of ageing. This is not the first time that we obtain data that apparently contradict the theory. In a previous study, we compared mitochondrial membrane lipids from whole Nothobranchius rachovii (MLSP $=14$ months) and Nothobranchius furzeri (MLSP $=7$ months), which resulted from two separate experiments (LucasSanchez et al. 2014; Almaida-Pagán et al. 2019), and showed that the shorter-lived species had the lowest PIn values. Afterwards, lipid profiles from whole $N$. korthausae, $N$. rachovii, $N$. guentheri and Aphyosemion australe kept under the same feeding and housing conditions were correlated with the MLSP of each species (MLSP $=80,63,53$ and 156 weeks, respectively) (de Costa et al. 2020). Results showed a negative correlation between membranes total PIn and fish MLSP, meaning that the most long-lived Nothobranchius species have 
a lower susceptibility to oxidative damage, which was in accordance with the LHA theory of ageing. However, the magnitude of the observed decrease in PIn associated with every doubling of MLSP was of only $2 \%$, which may indicate that specific tissues contribute more than others to this relationship, the inter-tissue differences masking the overall PIn when the whole fish is analysed.

In the present study and the case of $C$. viridis, Chromis fishes are considered a priori a model for short-lived reef inhabitants (Wantiez and Thollot P. 2001). Although they belong to a different genus, $C$. viridis share a similar habitat and feeding behaviour with clownfish. The main difference between them is that $C$. viridis undergo severe predation in the post-settlement phase (Hixon and Carr 1997) and have high juvenile and adult mortality. This, combined with a very rapid growth $(80 \%$ of maximum size reached within the first year), clearly indicates that these animals are short-lived in the wild, and therefore, tissues with more unsaturated membranes should be expected in order to cope with the LHA theory of ageing.

In conclusion, the present study showed differences in membrane composition (phospholipid class and fatty acid compositions) among fish tissues that point to the importance of particular PLs for tissue-specific functions. Significant differences in liver, skeletal muscle and brain membranes among A. percula, A. clarkii and $C$. viridis were found. When only the two Amphiprion species were compared, results pointed to the existence of a negative relationship between membrane PIn value and lifespan, as it has previously been shown in mammals, birds and fish species of genus Nothobranchius. Nevertheless, when the two Amphiprion species were compared to the shorter-lived $C$. viridis, data contradicted what the LHA theory of ageing posits. Although new studies including a wider number of anemonefish and other phylogenetically related species with different MLSP should be carried out to reinforce what was found in the present work, this data along with those obtained in previous studies on fish denote that the magnitude (and sometimes the direction) of the differences observed in membrane lipid composition and peroxidation index with maximum lifespan cannot explain alone the diversity in longevity found among fishes.
Author contribution Conceptualization: P.F.A.-P., A.C., P.M. and J.d.C.; methodology: P.F.A.-P. and A.L.; formal analysis: P.F.A.-P.; investigation: P.F.A.-P.; resources: P.F.A.-P.; writing - original draft: P.F.A.-P.; writing—review and editing: A.M.N., E.T., M.A.R.d.L., A.C. and J.d.C.; supervision: A.C. and J.d.C.; funding acquisition: P.F.A.-P., M.A.R.d.L. and J.d.C.

Funding Open Access funding provided thanks to the CRUE-CSIC agreement with Springer Nature. This work was supported by Fundación ONCE (research grant to P.F.A.-P as part of the "Oportunidad al Talento" Programme for disabled scientists, co-financed by ESF), the Spanish Ministry of Economy and Competitiveness, the Instituto de Salud Carlos III through CIBERFES (CB16/10/00239), the Séneca Foundation through grant 19899/GERM/15 and the Spanish Ministry of Science, Innovation and Universities (RTI2018-093528-B-I00), all of them co-financed by FEDER.

Data availability The datasets generated during and/or analysed during the current study are available from the corresponding author on reasonable request.

Code availability Not applicable.

\section{Declarations}

Ethics approval Fish were treated in accordance with the current Spanish law regarding animal's experiments, and the experimental protocol performed for this work was approved by the Bioethics Committee of the University of Murcia (A13160603, from the Consejeria de Agua, Agricultura, Ganaderia y Pesca, Comunidad Autonoma de la Region de Murcia, Spain).

Consent to participate Not applicable.

Conflict of interest The authors declare no competing interests.

Open Access This article is licensed under a Creative Commons Attribution 4.0 International License, which permits use, sharing, adaptation, distribution and reproduction in any medium or format, as long as you give appropriate credit to the original author(s) and the source, provide a link to the Creative Commons licence, and indicate if changes were made. The images or other third party material in this article are included in the article's Creative Commons licence, unless indicated otherwise in a credit line to the material. If material is not included in the article's Creative Commons licence and your intended use is not permitted by statutory regulation or exceeds the permitted use, you will need to obtain permission directly from the copyright holder. To view a copy of this licence, visit http://creativecommons.org/licenses/by/4.0/. 


\section{References}

Aldenhoven J (1986) Local variation in mortality rates and life-expectancy estimates of the coral reef fish Centropyge bicolor (Pisces: Pomacanthidae). Mar Biol 92:237-244

Almaida-Pagán P, Ortega-Sabater C, Lucas-Sánchez A et al (2019) Age-related changes in mitochondrial membrane composition of Nothobranchius furzeri.: comparison with a longer-living Nothobranchius species. Biogerontology 20:83-92

Almaida-Pagán PF, de Costa J, Mendiola P, Tocher DR (2012a) Age-related changes in mitochondrial membrane composition of rainbow trout (Oncorhynchus mykiss) heart and brain. Comp Biochem Physiol - B Biochem Mol Biol 163:129-137

Almaida-Pagán PF, de Costa J, Mendiola P, Tocher DR (2012b) Changes in tissue and mitochondrial membrane composition during rapid growth, maturation and aging in rainbow trout, Oncorhynchus mykiss. Comp Biochem Physiol - B Biochem Mol Biol 161:404-412

Barja G (2013) Updating the mitochondrial free radical theory of aging: an integrated view, key aspects and confounding concepts. Antioxid Redox Signal 19:1420-1445

Barja G (2019) Towards a unified mechanistic theory of aging. Exp Gerontol 124:110627

Barja G (2004) Free radicals and aging. Trends in Neurosci 27:595-600

Barja G, Herrero A (1998) Localization at complex I and mechanism of the higher free radical production of brain nonsynaptic mitochondria in the short-lived rat than in the longevous pigeon. J Bioenerg Biomembr 30:235-243

Bielski BJH, Arudi RL, Sutherland MW (1983) A study of the reactivity of H02/02- with unsaturated fatty acids. J Biol Chem 258:4789-4761

Blanco M, Sherman P (2005) Maximum longevities of chemically protected and non-protected fishes, reptiles, and amphibians support evolutionary hypotheses of aging. Blanco MA, Sherman PW Mech Ageing Dev 2005; 126(67)794-803 126:794-803

Buston P (2003) Mortality is associated with social rank in the clown anemonefish (Amphiprion percula). Mar Biol 143:811-815

Buston P, García M (2007) An extraordinary life span estimate for the clown anemonefish Amphiprion percula. J Fish Biol 70:1710-1719

Chaudhary AK, Nokubo M, Reddy GR et al (1994) Detection of endogenous malondialdehyde- deoxyguanosine adducts in human liver. Science (-80) 265:1580-1582

Christie WW (2003) Lipid analysis: isolation, separation, identification and structural analysis of lipids, 3rd edn. Oily Press, Somerset, UK

Csiszar A, Podlutsky A, Podlutskaya N et al (2012) Testing the oxidative stress hypothesis of aging in primate fibroblasts: is there a correlation between species longevity and cellular ROS production? J Gerontol A Biol Sci Med Sci 67:841-852

De Costa J, Barja G, Almaida-Pagan PF (2020) Membrane peroxidation index and maximum lifespan are negatively correlated in fish of genus Nothobranchius. J Exp Biol 223:jeb224003
Eckert G (1987) Estimates of adult and juvenile mortality for labrid fishes at one tree reef, great barrier reef. Mar Biol 95:161-171

Elliot J, Elliot J, Mariscal R (1995) Host selection, location, and association behaviors of anemonefishes in field settlement experiments. Mar Biol 1995;122(3)377-89 122:377-389

Feng Y, He D, Yao Z, Klionsky DJ (2014) The machinery of macroautophagy. Cell Res 24:24-41

Folch J, Lees M, Stanley S (1957) A simple method for the isolation and purification of total lipides from animal tissues. J Biol Chem 226:497-509

Garcia-Herrera N, Ferse SCA, Kunzmann A, Genin A (2017) Mutualistic damselfish induce higher photosynthetic rates in their host coral. J Exp Biol 220:1803-1811

Herrero A, Barja G (1998) H 2 O 2 production of heart mitochondria and aging rate are slower in canaries and parakeets than in mice : sites of free radical generation and mechanisms involved. Mech Ageing Dev 103:133-146

Hixon M, Carr M (1997) Synergistic predation, density dependence, and population regulation in marine fish. Science 277:946-949

Hulbert AJ (2005) On the importance of fatty acid composition of membranes for aging. J Theor Biol 234:277-288

Hulbert AJ, Faulks SC, Buffenstein R (2006a) Peroxidationresistant membranes can explain longevity of longest-living rodent. J Gerontol 61:1009-1018

Hulbert AJ, Pamplona R, Buffenstein R, Buttemer WA (2007) Life and death: metabolic rate, membrane composition, and life span of animals. Physiol Rev 87:1175-1213

Hulbert AJ, Turner N, Hinde J, Else P (2006b) How might you compare mitochondria from different tissues and different species? J Comp Physiol B 176:93-105

Ku H, Brunk U, Sohal R (1993) Relationship between mitochondrial superoxide and hydrogen peroxide production and longevity of mammalian species. Free Rad Biol Med 15:621-627

Lambert AJ, Boysen HM, Julie A et al (2007) Low rates of hydrogen peroxide production by isolated heart mitochondria associate with long maximum lifespan in vertebrate homeotherms. Aging Cell 6:607-618

López-Lluch G, Santos-Ocaña C, Sánchez-Alcázar J, Al E (2015) Mitochondrial responsibility in ageing process : innocent, suspect or guilty. Biogerontol 16:599-620

Lucas-Sánchez A, Almaida-Pagán PF, Mendiola P, de Costa J (2014) Nothobranchius as a model for aging studies. A review. Aging Dis 5:

Lucas-Sanchez A, Almaida-Pagan PF, Tocher DR et al (2014) Age-related changes in mitochondrial membrane composition of Nothobranchius rachovii. Journals Gerontol Ser A Biol Sci Med Sci 69A:142-151

Mariscal R (1970) The nature of the symbiosis between indoPacific anemone fishes and sea anemones. Mar Biol 6:58-65

Miwa S, Jow H, Baty K et al (2014) Low abundance of the matrix arm of complex I in mitochondria predicts longevity in mice. Nat Commun 5:3837

Modi HR, Katyare SS, Patel MA (2008) Ageing-induced alterations in lipid/phospholipid profiles of rat brain and liver mitochondria: implications for mitochondrial energylinked functions. J Membr Biol 221:51-60 
Moyer J (1986) Longevity of the anemonefish Amphiprion clarkii at Miyake-Jima, Japan with notes on four other species author ( $\mathrm{s}$ ): Jack T . Moyer Published by : American Society of Ichthyologists and Herpetologists ( ASIH ) Stable URL: http://www.jstor.org/stable/144. Copeia 1986:135-139

Naudí A, Jové M, Ayala V, et al (2013) Membrane lipid unsaturation as physiological adaptation to animal longevity. Front Physiol 4 DEC:1-13

Olsen RE, Henderson RJ (1989) The rapid analysis of neutral and polar marine lipids using double-development HPTLC and scanning densitometry. J Exp Mar Bio Ecol 129:189-197

Pamplona R, Portero-Ortín M, Riba D, Requena JR, Thorpe SR, López-Torres M, Barja G (2000) Low fatty acid unsaturation: a mechanism for lowered lipoperoxidative modification of tissue proteins in mammalian species with long life span. J Gerontol A Biol Sci Med Sci 55A:B286-B289

Pamplona R, PorteroOrtín M, Ruiz C, Prat J, Bellmunt MJ, Barja G (1998) Mitochondrial membrane peroxidizability index is inversely related to maximum life span in mammals. J Lipid Res 39:1989-94

Pamplona R (2008) Membrane phospholipids, lipoxidative damage and molecular integrity: a causal role in aging and longevity. Biochim Biophys Acta 1777:1249-1262

Pamplona R, Barja G, Portero-Otin M (2002) Membrane fatty acid unsaturation, protection against oxidative stress, and maximum life span: a homeoviscous-longevity adaptation? Ann N Y Acad Sci 959:475-490

Paradies G, Ruggiero FM, Quagliariello E (1992) Age-dependent changes in the activity of anion carriers and in the lipid composition in rat heart mitochondria. Ann N Y Acad Sci 673:160-164

Sahm A, Almaida-Pagán P, Bens M et al (2019) Analysis of the coding sequences of clownfish reveals molecular convergence in the evolution of lifespan. BMC Evol Biol 19:89

Shen E, Song C, Lin Y et al (2014) Mitoflash frequency in early adulthood predicts lifespan in Caenorhabditis elegans. Nature 508:128-132

Tozzini E, Dorn A, Ng'oma E et al (2013) Parallel evolution of senescence in annual fishes in response to extrinsic mortality. BMC Evol Biol 13:77

Wantiez L, Thollot P (2001) Settlement, post-settlement mortality and growth of the damselfish Chromis fumea (Pisces: Pomacentridae) on two artificial reefs in New Caledonia (south-West Pacific Ocean). WJ Mar Biol Assoc U K 80:1111-1118

Witting L, Horwitt M (1964) Effect of degree of fatty acid unsaturation in tocopherol deficiency-induced creatinuria. J Nutr 82:19-33

Zsurka G, Peeva V, Kotlyar A, Kunz W (2018) Is there still any role for oxidative stress in mitochondrial DNA-dependent aging? Genes (basel) 9:175

Publisher's note Springer Nature remains neutral with regard to jurisdictional claims in published maps and institutional affiliations. 